\title{
Real-Time Traffic Sign Recognition System based on Colour Image Segmentation
}

\author{
Vishal R. Deshmukh \\ Research Scholar \\ Computer Engineering \\ Department \\ S.S.B.T. College of \\ Engineering And Technology \\ Jalgoan, Maharastra, India
}

\author{
G. K. Patnaik, Ph.D \\ Head Of Department \\ Computer Engineering \\ Department \\ S.S.B.T. College of \\ Engineering And Technology \\ Jalgoan, Maharastra, India
}

\author{
M. E. Patil \\ Associate Professor \\ Computer Engineering \\ Department \\ S.S.B.T. College of \\ Engineering And Technology \\ Jalgoan, Maharastra, India
}

\begin{abstract}
The traffic symbol system has been studies for many years. The system mainly has two phases, 1) Sign detection, 2) Recognition. It is one of the most important research area for enabling vehicle driving assistances. The automatic driving system should be simple in order to detect symbol with high responses. The challenge gets more difficult in order to make system simple while avoiding complex image processing techniques to detect symbol. The propose system consist of three main phases, 1) Frame selection, 2) Symbol detection, 3) Symbol Recognition. In frame selection phase the best frame possibly consisting of traffic symbol is selected from number of frames. In detection phase color image segmentation is perform on given frame in order detect the symbol. In recognition phase the detected traffic symbol is recognized using joint tranceform correlation (JTC) technique which is generally use for pattern matching.
\end{abstract}

\section{General Terms}

Vehicle, road sign, detection, color, techniques

\section{Keywords}

Segmentation, region of interest, detection and recognition, edge base, Joint Transform Correlation technique

\section{INTRODUCTION}

The system is develop to first detect, and then recognize a set of Traffic sign from within frame. Such a system should be capable to studies the traffic scene image captured by the camera, obtain the traffic symbol region and make smart decisions. Generally, the system working of traffic symbol detection and recognition is divided into three stages: frame selection, segmentation and detection, recognition. Detection stage is accountable for finding region of interest (ROI) from the picture frame that is taken by a video camera mounted on top of a vehicle. ROI is the most probable part of the image that may contain traffic symbols. The second stage of automatic detection and recognition of traffic symbol. In this stage each ROI is according to their edge. In the last stage individual traffic symbol is recognized from its class. Detection algorithm can be based on template properties of traffic symbols. However, color information of input images is sensitive to the change of lighting conditions and weather. Moreover city like environment contains lots of non-symbol objects with shape and color information similar to the traffic symbols. It can increase number of symbol-candidates and increase recognition time and number of false-positive recognitions - then non-symbol object is recognized as a traffic symbol. The system will first see about those techniques that are based on finding object regions in color images. It will also see couple of techniques that deal with color segmentation to highlight how this has been used for outdoor scene analysis.

\subsection{Challenges}

There are many problems that system has to take into account as the different conditions, blur image from moving vehicle, displacement of the traffic symbols, multiple traffic symbols blocking each other, faded traffic symbols due to effect of whether obstruction of traffic symbols by either natural or artificial elements like trees or street posters and the presence of objects having similar type of shape and color as traffic symbols.

\section{RELATED WORK}

The region comes near attempt to single out areas of images that are uniform matching to a given set of characteristics. Given areas may be develop, shrivel, merged, split, created or destroyed during the segmentation process. There are two typical region-based segmentation algorithms: regiongrowing, and split-and-merge. Region growing(develop) is one of the most simple and popular algorithms and it starts by selecting a starting point or seed pixel. Then, the region develop by adding neighboring pixels that are uniform, according to a certain match criterion, increasing step by step the size of the region. Typical split-and-merge techniques consist of two basic steps. If this region does not follow with a uniform criterion the region is split into four quadrants and each quadrant is tested in the same way until every square region created in this way contains homogeneous pixels. Next, in a second step, all adjacent regions with similar attributes may be merged following other criteria. There are many options used by the system for detection and recognition of traffic symbols from the natural image. Mainly the system consist of detection phase and classification phase. The detection phase widely uses the image segmentation. When working with the gray-level images, the search is mainly based on the shape and can be quite computationally expensive. For traffic symbol detection, several methods have been developed based on shape recognition. T. Ueta and Y. Sumi and N. Yabuki and S Matsumae[10] used a selforganizing map (SOM) to extract a contour line and recognize the traffic-symbol shape from it.R. Belaroussi and J. Tarel[23] proposed a geometric model of the image gradient orientation to detect triangular symbols. R. Marmo and L. Lombardi [21] used optical flow analysis to identify the rectangular symbols and then by searching gray-level discontinuity on the image and Hough transform for detection of Milepost symbols. Loy and Barnes [22] implemented an algorithm based on fast radial symmetry, where patterns of edge orientations are exploited to detect triangular, square, and octagonal traffic symbols. However, gradient based 
feature detection is, by nature, sensitive to noise, and many shape detectors are slow in computing over large images.

Color images are more complex than gray scale images as instead of a single intensity value for a pixel, each pixel is usually denoted by three component values as red, green and blue. Clustering based methods are ideal to use for segmentation of color images. Clustering algorithms can be either hierarchical or partitional. Hierarchical techniques involve the clusters themselves being classified into groups, where the process is repeated at different levels to form a tree. Partitional techniques forms clusters by optimizing a clustering criterion, where the classes are mutually exclusive thus forming a partition of the data Statistical clustering is the most common form of clustering, which involves assigning each occurrence of a particular color to one particular cluster regardless of whether each pixel having the color are located near to each other spatially in the image, in which case these pixels would not form a valid segment from the visual point of view. C. Bahlmann and Y. Zhu and R. Visvanathan and M. Pellkofer and T. Koehler [16] detected symbols using a set of color-sensitive Haar wavelet features obtained from AdaBoost training and temporal information propagation. Classification was performed using Bayesian generative modeling with temporal hypothesis fusion. X. Baro and S. Escalera and J. Vitri and O. Pujol and P. Radeva [9] proposed a novel binary classifier through an evolutionary version of AdaBoost. To avoid the limitations of the original boosted classifiers in terms of the dimensionality of the feature space. A. Ruta and Y. Li and X. Liu[2] used an image representation and discriminative feature selection method for traffic-symbol recognition.

The classifiers based on the image features have a major drawback that, due to the exhaustive search over the feature set, the training time grows with respect to the number of features. Color-based symbol detection relies on color, whereas the grayscale method concentrates on the geometry/shape of the object. Recent works have used both color segmentation and shape recognition to improve the detection rates. First, candidate regions are selected using color features, and then, an edge based method is employed to the perimeter of the regions for the detection step. X. Chen and J. Yang and J. Zhang and A. Waibel [14] presented an approach to detect and recognize Chinese symbols and translated the recognized text into English. The technique embeds multi resolution and multi scale edge detection, adaptive searching, color analysis, and affine rectification in a hierarchical framework to handle text in different sizes, orientations, color distributions, and backgrounds. King Hann Lim, Li-Minn Ang, Kah Phooi Seng[5] discussed new hybrid technique which proposed for traffic sign detection. This system is combination of knowledge based analysis and radial basis function neural classifier (RBFNN). Firstly the traffic signs are detected from the natural image using color image segmentation technique. The extracted signs are then passed to the recognition system for classification. The recognition system consists of three stages: color histogram classification, shape classification and RBF neural classification. Unique color and shape of traffic signs are used to classify them into smaller subclass and then can be easily recognized using RBFNN. In this system traffic sign features are from the image using principle component analysis. Then the most discriminant features are obtained using the Fisher's Linear Discriminant (FLD). Siti Sarah Md Sallah, Fawnizu Azmadi Hussain, and Mohd Zuki Yusoff [24] proposed new method for road sign detection and recognition algorithm for an embedded application. The algorithm uses Hue Saturation
Intensity (HSI) color space to segment and locate the region of interest (ROI) and determines the shape of the road sign(diamond, square, hexagonal and circular ) in natural images. The shape is then used to classify road symbols in four categories i.e. warning, mandatory, prohibitory and informational signs. Here the characteristics of the shapes such as area and perimeter variables are used to identify the symbols in road signs. These variables are compared with the template library. Madhusudan Joshi, Mohan Jeet Singh and Saurabh Dalela [4] presented colored traffic detection using correlation techniques based on joint transform correlation (JTC). In this paper, author proposes the application of multichannel correlation for automatic colored traffic sign detection. Mohammad S. Alam and C. N. Wai [3] proposed the Fringe-Adjusted Joint Transform Correlation technique (FJTC). Fringe-Adjusted JTC is one that gives better correlation performance than JTC for target detection. In FJTC, the joint power spectrum (JPS) is multiplied by the filter called Fringe-adjusted filter (FAF) before applying the IFT. Vavili Andrey and Kang Hyun Jo [6] proposed the detection and recognition algorithm for road signs of different category. As color and shape of traffic symbol provides vital information, they used the RGB color space to detect the symbol. Again to fight with changing conditions due to lighting and weather such as dark illumination, rainy and foggy weather, two restriction rules are used. First rule is bounding constraints for each color component which provides good detection result in images in good lighting condition. Second rule is using normalized color information and allows sign detection in dark images. Shape of information sign is different from the shape of warning and restricting sign. So the recognition process used here depends upon the shape of sign. Sign candidate is extracted from image and classified it as circle or triangle using background shape histograms. Then inner part of the mask is converted into binary mask and template matching algorithm is used for final recognition. Particularly in this paper, author tries to find out the inner meaningful part with the help of template matching. Auranuch Lorsakul and Suthakurn [15] proposesd Traffic Sign Recognition (TSR) in which first images are preprocessed with several image processing techniques such as threshold techniques, Gaussian filter, canny edge detection and then neural network is used to recognize the traffic sign patterns. Jung Chieh Hsien, Yi-Sheng Liou and Shu Yuan Chen [18] proposed a novel road sign detection and recognition method. In this projection technique is used to determine the position of the road sign in image. Then the Hidden Markov model is used to match the detected road sign with the image in the database. In this color images in RGB color space are first converted into HSV color space and then quantized into specific colors. The vertical and horizontal projections of whole images in the specific colors existing in road sign are used to detect the positions of the road signs. The recognition stage uses only the local features around the detected positions. The horizontal and vertical projections of the background in the local area are used to remove irrelevant road signs. The candidate road signs are then sorted using Hidden Markov model. The one with the first rank is considered as the recognition result. Xiaohong Gao, Kunbin Hong, Peter Passmore, Lubov Podladchiko and Dmity Shaposhnikov [1] used new approach for segmenting traffic signs from the image using the CIECAM, a standard color appearance model. Color is the most dominant features in retrieving the road symbols from the natural image. Due to change of whether conditions, such as sunny, cloudy and rainy, the color of the traffic may appear different. The color spaces such as HIS, RGB, L*a*b* are normally limited to 
only one lighting conditions. But the CIECAM color appearance model outperforms in other color space in all conditions. Tang Jin, Liang Xiong, Xie Bin, Chen Fangyan [8] proposesd a method which combines color and shape to detect the traffic symbol. In this Hue and saturation are used to detect the red color of the sign. Circles are detected through the improved round- degree method. An improved Kalman filter is used to track multiple targets in the next frames. For recognition of traffic symbol, the feature extraction method based on the two dimensional Principal Component Analysis (2DPCA) is proposed. Miguel Angel Garcia -Garrido, Miguel Angel Sotelo and Ernesto Martin -Gorostiza [19] proposed a system which consists of three stages: first, detection using Hough transforms to get the information about edges of the image; second classification using neural network and final stage is tacking which uses the Kalman filter. Y. Nguwi and A. Kouzani [13] proposed a system which consists of two modules: detection and classification. The detection module segments the image using HIS color space and locates the traffic symbol. The classification module determines the type of detected traffic symbol using a series of one to one architectural multi layer perceptron neural networks. Hua Huang, Chao Chen, Yulan Jia, and Shuming Tang [12] proposed two stage automatic detection and recognition algorithm. In first stage, color segmentation and Hough transform are used for ROI detection. Considering the difference between the actual road symbol and the standard road symbols, the pictograph on road symbols is extracted for recognition. Adaptive Hausdorff distance based on similarity weighting is used in recognition stage. Ian Sebanja, D. B. Megherbi [11] proposesd a multi-layered hierarchical scheme containing three phases. Traffic symbol color segmentation, shape recognition and classification. The system is robust and invariant to rotation, scaling and image translation. The traffic symbol shape detection and traffic symbol recognition and classification are both based on principal component analysis (PCA). Jitendra N. Chourasia, Preet Bajaj [7] proposesd an algorithm that uses the $\mathrm{YCbCr}$ color space for color image segmentation. The extraction and detection of traffic symbol is using color of the traffic symbol. The sign is extracted by considering the maximum distance of the pixels from centroid. The minimum Euclidean distance classifier is used to detect the shape of the symbol. Perceptron neural network is used to recognize the traffic symbol. Woong-Jae Won, Sungmoon Jeong, and Minho Lee [25] proposesd new preprocessing method for detecting traffic symbol based on visual salience map model. As the traffic symbols have dominant color contrast against environment, color information with center surround difference normalization is used as an input feature extraction to reduce the noise influence as well as intensify the color characteristics. Edge feature map is used to reflect the shape characteristics of traffic symbol. The weighted sum of color feature map and edge feature map finally constructs traffic symbol saliency map. The system that detects candidate regions as maximally stable external regions (MSERs), which offers robustness to the various lightning conditions. Recognition is based on cascade of support vector machine (SVM) classifiers that were trained using histogram oriented gradient (HOG) features. A new two stage method. Firstly, two way integration method including both methods drived by task and methods drived by data is used for segmentation, which improves accuracy of detection. And then edge extraction and morphological operations are used to obtain enclosed area. The shape of enclosed area is determined by the circularity and central double cross shape measurement in order to detect the prohibition signs.
The proposed system consist of three phases : i) frame selection ii) detection and iii) recognition. Detection phase consist of image segmentation using edge detection and recognition Joint Transform Correlator for pattern matching.

\section{PROPOSED SYSTEM}

The workflow of the system real traffic symbol detection and recognition system which is proposed in this paper is shows in Fig. 1. The traffic symbol detection and recognition system, it is detects the traffic symbol using the color feature. The detection phase is initiated by performing color channel separation which enhances the color separation of an image with band to band correlation and segmentation of color image using edge detection technique. The segmented regions are then verified using the possible ROI and filter the highly possible traffic symbols. Afterwards the traffic symbol recognized by using joint transform correlation method which is carried out to recognize the traffic symbols from segmented image[26].

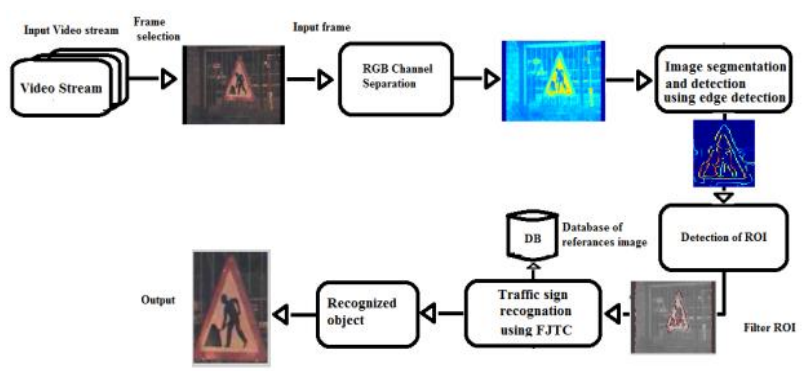

Fig 1 : Workflow of propose system

\subsection{Frame Selection}

The proposes real time traffic sign recognition system uses video stream captured by web camera, with frame resolution of 30fps. Vedio stream obtained from web camera is converted in .avi format. Each frame is of size 320x240. after selecting good frame which may likely have candidate object is resized as $160 \times 160$.

\subsection{Segmentation Techniques}

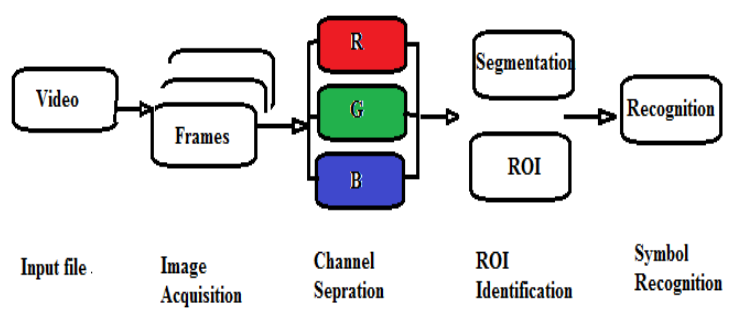

Fig 2 : General architecture for Real time traffic sign recognition

The proposed system uses RGB colour scheme because the camera used provides RGB images which shown in fig. 2. When using RGB to perform color segmentation, there is a need to separate the original image into 3 different images each representing one of the color channels that make up the image namely, red, green and blue in fig. 3. These new color channel images, are then processed further to perform color segmentation[26].

Firstly, image prepossessing techniques are used for smoothing and noise-removal. Second, the edge representation is generated. Differentiation is done to find the edge-strength at each point in the image. Separation is done unwanted edges. 
Lastly, the reaming edges are joined to form line segments and features are computed.

Edge based segmentation is used for detecting location of pixels in the image that correspond to the boundaries of the objects seen in the image. It is then assumed that since it is a boundary of a region or an object then it is closed and that the number of objects of interest is equal to the number of boundaries in an image. For precision of the segmentation, the perimeter of the boundaries detected must be approximately equal to that of the object in the input image. In this proposed a set of algorithm used to perform segmentation of captured frames through boundary analysis as well as it perform region of interest (ROI). ROI is find out on basis of segmentated image[2].

\subsection{Sign Recognition}

The system uses Joint Transform Correlator (JTC) technique for pattern recognition. The correlation process implements by JTC. In correlation images are placed side by side in plane to take Fourier transform (FT). Then, The intensity of this Fourier Transform called joint power spectrum (JPS) is calculated. The output of the segmentation is the intensity of the FT of JPS. If the reference and the target images are represented as $r(x, y) \& t(x, y)$, and are separated by $2 x_{0}$, then the input plane is

$i(x, y)=r\left(x-x_{0}\right)+t\left(x+x_{0}\right)$

If $R(a, b)$ and $T(a, b)$ are FT of reference and the target respectively, with $a \& b$ scaled by $1 / \lambda f$ (where $\lambda$ is the wavelength of light and $f$ is the focal of the lens), the JPS is

$I(a, b)=$

$|R(a, b)|^{2}+|T(a, b)|^{2}+2 R(a, b) T(a, b) \cos \left(a x_{0}\right)-(2)$

And finally the output is obtained as FT of JPS and is proportional to:

$r(x, y) \otimes r(x, y)^{*}+t(x, y) \otimes t(x, y)^{*}+r\left(x-x_{0}, y\right)^{*} \otimes$

$t\left(x+x_{0}\right)+t\left(x+x_{0}, y\right)^{*} \otimes r\left(x-x_{0}, y\right)(3)$

where, $\otimes$ denotes correlation and $*$ denotes complex conjugate. In JTC the cross correlation peaks are obtain only if the references and target image both are same, else no cross correlation peaks will obtain. [4].

\section{EXPERIMENTAL RESULTS}

The traffic symbol recognition system must be accurate and fast enough to recognize the traffic symbol in order to avoid the upcoming hazards. So to evaluate the performance of the proposed system, number of images tested. The collection of test and reference stream video mostly obtained from the Google and YouTube. Fig. 3. shows the representative sample of these images. The segmentation, detection and recognition results obtained from the proposed method are shown in fig. 1. In Fig. 3. show input frame which is selected as best frame, Fig. 4. show RGB Channel separation, Fig. 5. Segmented Image by Using Edge Base, Fig. 6. show region of interest, Fig. 7. show traffic sign recognition, Fig. 9. show graph of Joint Transform Correlator.

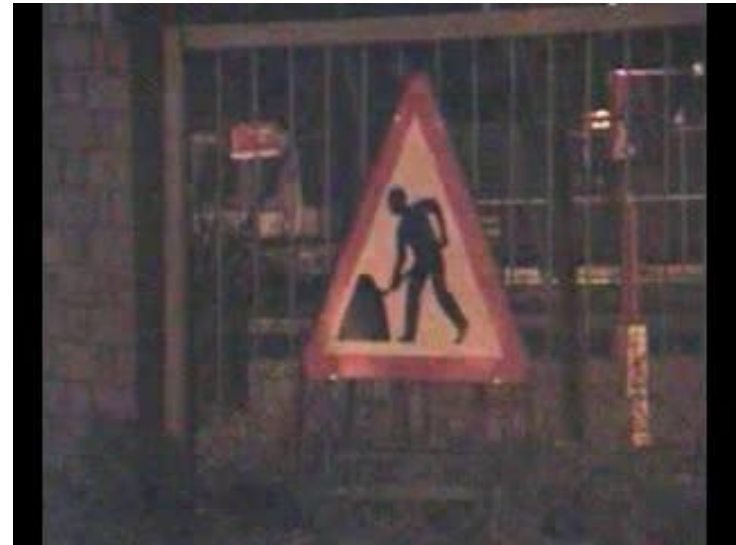

Fig 3: Frame from video string

The video file extract in the form of number of frames. In this collection of frame we are trying to select that frame, which contain traffic sign. This frame hand over for color channel separation. The fig. 3. shows frame.

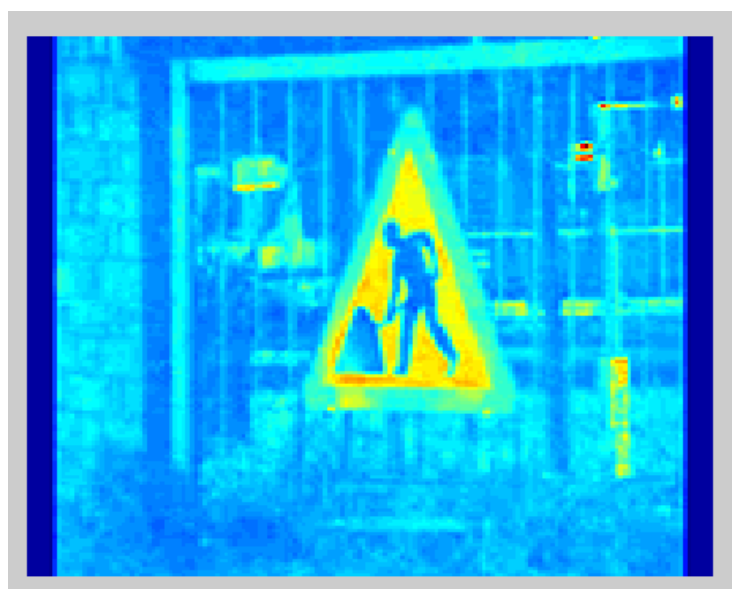

Fig 4: RGB Channel Separation Image.

Color channel Separate the image into 3 colour channels i.e. Red, Green and Blue. Divide the pixels from one colour channel with pixels of different colour channel which is shown in Fig. 4.

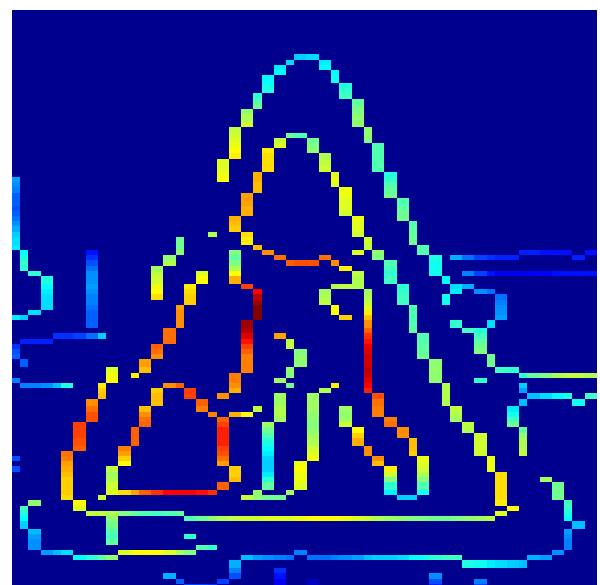

Fig 5: Segmented Image by Using Edge Base

In fig. 5. show segmented part of color image, which is very important in system.

In Fig. 6. show region of interest which find out from given frame which is segmented on the basis of edge base. 


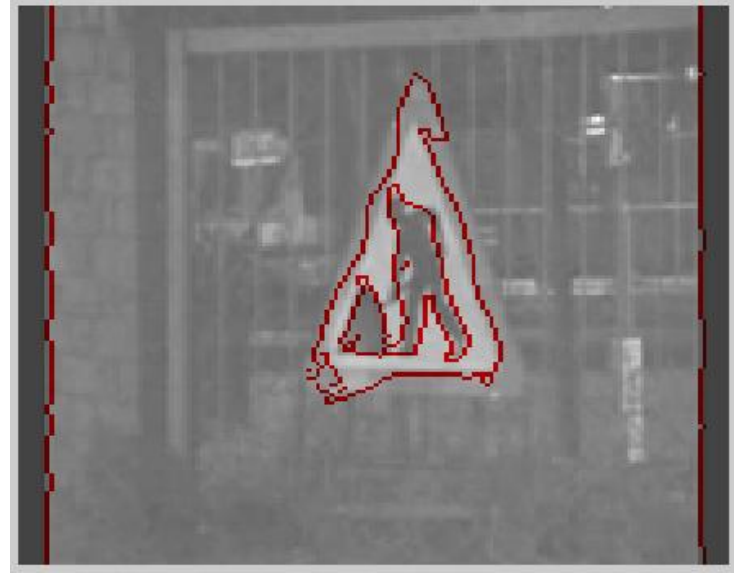

Fig 6: Region of Interest

After we getting the region of interest it is match with database image with help of joint transform correlate. This actually third stage which performing traffic sign recognition. This recognition create graph which is shown in Fig. 9. But the out of the system is shown in fig. 7 .

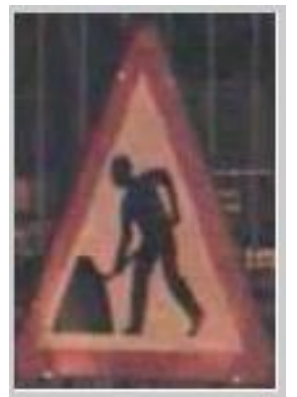

Fig 7: Output of Recognition phase

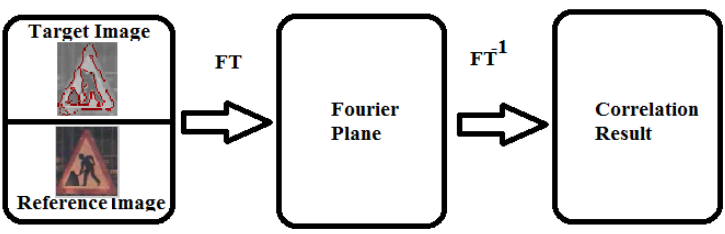

Fig 8: Working of Joint Transform Correlator.

fig. 8. is actually show the working of joint transform correlator technique. It is take two image for pattern matching, one is target image with ROI and second is references image which is store in database, if they are match the JTC graph is generate in fig. 9 .

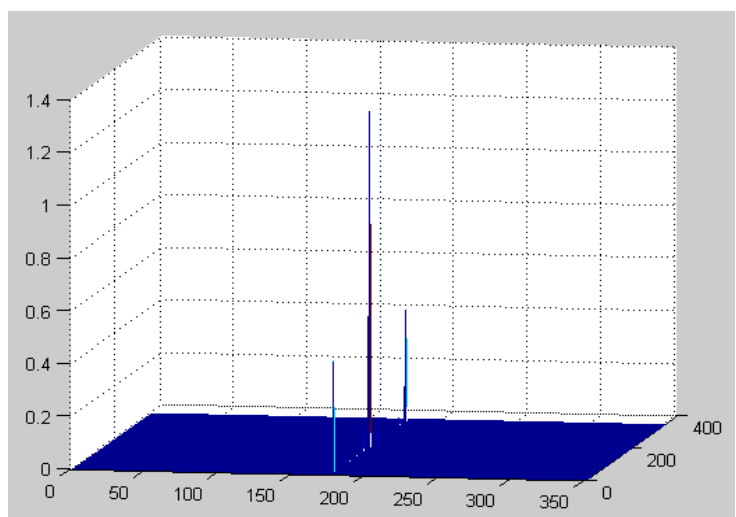

Fig. 9:Graphical output of Joint Transform Correlator
Table 1. Result of Final Recognition Stage

\begin{tabular}{|l|c|}
\hline Total number of images & 78 \\
\hline Total number of signs & 43 \\
\hline Detection of traffic sign & 40 \\
\hline False detection & 3 \\
\hline
\end{tabular}

In this project we are testing 78 traffic sing which are provided in the form of video file that is number of frames. To evaluate the performance of the proposed traffic symbol recognition system, the images containing the traffic symbol taken in different lighting conditions are tested. Table - I show the results obtained from the final stage of the traffic symbol recognition system. The number of image tested on the proposed system are 78 which contains total of 43 traffic symbol signs. Out of which 40 traffic symbols are recognized correctly, 3 traffic symbols are detected incorrectly.

\section{CONCLUSION}

The proposed system will be applicable in real time of modifications. The response time of proposed system will increase by the use of dedicated hardware and low level language like $\mathrm{C}$.

\section{REFERENCES}

[1]. X.W. Gao, L. Podladchikova, D. Shaposhnikov, K. Hong, and N. Shevtsova, Recognition of traffic symbols based on their colour and shape features extracted using human vision models, J. Vis. Commun. Image Represent, 17(4):675-685, Aug 2006.

[2]. A. Ruta and X. YongminLi,"Real-time traffic sign recognition from video by class specific discriminative features," Pattern Recognition, pp. 416\{430, 2010.

[3]. Mohammad S. Alam and C. N.Wai, Color pattern recognition using fringe -adjusted joint transform correlation, in Proc of IEEE, 2000.

[4]. Madhusudan Joshi, Mohan Jeet Singh, and Saurabha Dalela, Automatic colored traffic sign detection using optoelectronic correlation architectures, IEEE conference on Vehicular Electronics and Safety USA, Sept 22-24 2008.

[5]. K. H. Lim, Li-MinnAng, and K. P. Seng, 'New hybrid technique for traffic sign recognition," International Symposium on Intelligent Signal Processing and CommunicationvSystems (ISPACS2008) Bangkok, Thailand, 2008.

[6]. V. Andrey and J. Kang-Hyun. Automatic detection and recognition of traffic symbols using geometric structure analysis. in Proc. SICE-ICASE Int. Joint Conf, pages 1451-1456, 2006.

[7]. Jitendra N. Chourasia and Preet Bajaj, Centroid based detection algorithm for hybrid traffic sign recognition system, $3^{\text {rd }}$ International Conference on Emerging Trends in Engineeringand Technology, IEEE, 2010.

[8]. Tang Jin, Liang Xiong, Xie Bin, and Chen Fangyan. A method for traffic signs detection, tracking and recognition 5th International Conference on Computer Science and Education Hefei China, pages 24-27, August 2010.

[9]. X. Baro, S. Escalera, J. Vitri, O. Pujol, and P. Radeva, "Traffic symbol recognition using evolutionary adaboost detection and forest-ecoc classification," IEEE Trans. 
Intel. Trans. Syst, vol. 10, no. 1, pp. 113-126, March 2009.

[10].T. Ueta, Y. Sumi, N. Yabuki, and S. Matsumae, "A study on contour line and internal area extraction methd by using the self-organization map," in Proc. Int. Symp. Intel. Symbolal Process. Commun. Syst. Tottori Japan, pp. 685-688, 2006.

[11].Ian Sebanja and D. B. Megherbi. Automatic detection and recognition of traffic road signs for intelligent autonomous unmanned vehicles for urban surveillance and rescue in proc of IEEE, 2010

[12].Hua Huang, Chao Chen, Yulan Jia, and Shuming Tang, Automatic detection and recognition of circular road sign, in proc of IEEE, 2008.

[13].Y. Nguwi and A. Kouzani. Automatic traffic symbol recognition using neural networks. in Proc. Int. Joint Conf. Neural Netw., Vancouver, BC, Canada, July 16-21 2006.

[14].Y. Chang and X. Li, "Adaptive image region growing," IEEE Transactions on Image Processing, vol. 3, no. 6, pp. 868-873, 1994.

[15]. Auranuch Lorsakul and Jackrit Sauthakorn. Traffic sign recognition for intelligent vehical/driver assistance system using neural network on opencv. in 4th International Conference on Ubiquitous Robots and Ambient Intelligent (URAI), 2007.

[16].C. Bahlmann, Y. Zhu, R. Visvanathan, M. Pellkofer, and T. Koehler. A system for traffic symbol detection, tracking, and recognition using color, shape, and motion information. in Proc. IEEE Conf. Intel. Veh. Symp, pages 255-260, Jun 2005.

[17].H. Frigui and R. Krishnapuram, "A robust competitive clustering algorithm with application in computer vision," IEEE Transactions on Pattern Analysis and Machine Intelligence, vol. 21, no. 5, pp. 450-465, 1999.
[18].Jung-Chieh Hsien, Yi-Sheng Liou, and Shu-Yuan Chen. Road sign detection and recognition using hidden markov model in Asian Journal of health and Information Sciences, 1(1):85-100, 2006.

[19]. Miguel Angel Garcia Garrido, Miguel Angel Sotelo, and Ernesto Martin Gorostiza, Fast traffic sign detection and recognition under changing lighting conditions, in proc IEEE Intelligent Transportation Systems Conference, Toronto, Canada, Sept 17-20 2006.

[20].W. A. Perkins, "Area segmentation of images using edge points," IEEE Transactions on Pattern Recognition and Machine Intelligence, vol. 2, no. 1, pp. 8-15, 1980.

[21]. R. Marmo and L. Lombardi. Milepost symbol detection. in Proc. Int. Workshop Comput. Arch. Mach. Perception Sensing, pages 93-98, 2006.

[22].G. Loy and N. Barnes. Fast shape-based traffic symbol detection for a driver assistance system. in Proc. IEEE Conf. Int. Robots Syst., pages 70-75, 2004.

[23].R. Belaroussi and J. Tarel. Angle vertex and bisector geometric model for triangular traffic symbol detection. in Proc. Workshop Appl. Comput. Vis., pages 1-7, 2009.

[24].Siti Sarah Md Sallah, Fawnizu Azmadi Hussin, and Mohd Zuki Yusoff. Road sign detection and recognition system for real time embedded applications. International Conference on Electrical, Control and Computer Engineering, Pahang, Malaysia, June 21-22 2011.

[25].Woong-Jae Won, Sungmoon Jeong, and Minho Lee, Road traffic sign saliency map model, in Proc. Image and Vision Computing, New Zealand, pages 91-96, 2007.

[26].Z. T. Kardkovacs, Z. Paroczi, E. Varga, and A. S. P. Lucz, "Real-time traffic sign recognition system," IEEE-2011 International Conference on Intelligent Computation technology and Automation, 2011. 